\title{
Identification of most consistent and reliable anatomical landmark to locate and protect radial nerve during posterior approach to humerus: a cadaveric study
}

\author{
Apurba Patra $^{1}$, Priti Chaudhary ${ }^{1}$, Vishal Malhotra ${ }^{2}$, Kamal Arora $^{3}$ \\ ${ }^{1}$ Department of Anatomy, All India Institute of Medical Sciences, Bathinda (Pb), ${ }^{2}$ Department of Social and Preventive Medicine, Government Medical \\ College, Patiala, ${ }^{3}$ Department of Orthopedics, Government Medical College, Amritsar, India
}

\begin{abstract}
The location of the radial nerve (RN) is described with various bony landmarks, but such may be disturbed in the setting of fracture and dislocation of bone. Alternative soft tissue landmarks would be helpful to locate the nerve in such setting. To recognize certain anatomic landmarks to identify, locate and protect RN from any iatrogenic injury during surgical intervention such as open reduction and internal fixation. Forty arms belonging to 20 adult cadavers were used for this study. We measured the distance of RN from the point of confluence of triceps aponeurosis (TA), tip of the acromion and tip of the lateral epicondyle along the long axis of the humerus. These distances were correlated with the upper arm length (UAL). The average UAL was $32.64 \pm 0.64 \mathrm{~cm}$. The distance of the RN from the point of confluence of TA (tricepso-radial distance, TRD), tip of acromion (acromion-radial distance) and tip of lateral epicondyle of humerus (condylo-radial distance, CRD) was $3.59 \pm 0.16 \mathrm{~cm}, 14.27 \pm 0.59 \mathrm{~cm}$, and $17.14 \pm 1.29 \mathrm{~cm}$ respectively. No correlation was found with UAL. Statistically, TRD showed the least variability and CRD showed maximum variability. The minimum TRD was found to be $3.00 \mathrm{~cm}$. So this should be considered as the maximum permissible length of the triceps split. The point of confluence of the TA appears to be the most stable and reliable anatomic landmark for localization of the RN during the posterior approach to the humerus.
\end{abstract}

Key words: Radial nerve, Humerus, Acromion, Aponeurosis, Anatomic landmarks

Received April 2, 2020; Revised April 12, 2020; Accepted April 20, 2020

\section{Introduction}

The radial nerve (RN) arises from the posterior cord of the brachial plexus. The nerve crosses the spiral groove obliquely from medial to lateral over the posterior surface of the humerus. It then penetrates the lateral intramuscular

\section{Corresponding author:}

Kamal Arora (iD

Department of Orthopedics, Government Medical College, Amritsar 143001, India

E-mail:kka1967@gmail.com septum near the junction of the middle and distal thirds of the humerus $[1,2]$. The long tortuous course and close relation to the periosteum of humerus make RN most common peripheral nerve involved in upper extremity fracture [3]. RN palsies are mainly two types, Primary and secondary. Primary palsies are those mostly due to fracture humeral shaft and have been reported in $11.8 \%$ of all cases reported [4]. Secondary or iatrogenic palsies can occur during any kind of operative interventions for unstable humeral fractures, such as plating or open reduction and internal fixation. These iatrogenic palsies are not uncommon in routine practices and are of major concern [5] before planning intervention. Fracture humerus can be fixed through various surgical ap- 
proaches, such as anterior, anterolateral, posterior, modified posterior [6]. Although $\mathrm{RN}$ is at considerable risk in each of these approaches [7], posterior approach is considered to be the safest. So identification of the RN during posterior approach allows us to protect it during surgery and further aids in its localization in future revision surgery [8]. Some studies have established the anatomic relationship of the RN with various bony landmarks, such as medial epicondyle, lateral epicondyle of humerus and tip of the acromion [9-13]. Previously orthopaedic surgeons used to depend on these landmarks to identify the $\mathrm{RN}$ during surgical exploration of the humerus but the wide range of these proposed anatomic relationships may create difficulty to localize the RN during surgery. Moreover, such relationships with bony landmarks may not hold true in clinical settings, especially with fractures of the distal third of the humerus [14]. Therefore, we believe that surgeons need another consistent and reliable anatomic landmark to locate the RN more precisely during the 'triceps splitting' approach to the humerus.

With such a facile, the primary aim of our study was to measure the distance of the RN from the point of confluence of the triceps aponeurosis (TA) (soft tissue landmark), tip of acromion and tip of lateral epicondyle of humerus to locate the RN. Secondary aim was to define the most consistent and reliable anatomical landmark for intra operative identification of RN.

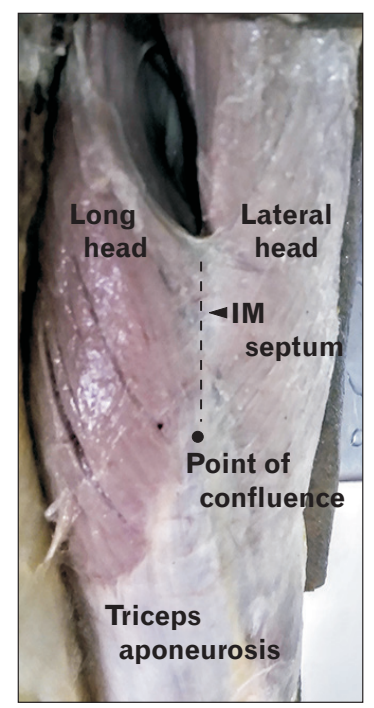

Fig. 1. Showing the point of intersection between the intramuscular (IM) septum (dotted line) with the triceps aponeurosis, labelled as 'point of confluence'.

\section{Materials and Methods}

The present study was done on 40 upper limbs belonging to 20 formalin embalmed cadavers ( 15 male, 5 female) aged between 45 to 60 years. Arms having any gross malformation or deformity were excluded from the study.The specimens were prepared through scapula-thoracic disarticulation and mid clavicular amputation. Each cadaveric upper extremity was placed at 90 degree flexion at elbow joint as this is the most common position used intraoperatively. A posterior midline skin incision starting from the tip of acromion up to the tip of subcutaneous olecranon was given. Subcutaneous tissue and deep fascia were also incised in the same line exposing the triceps muscle. The shiny TA was immediately visible on the posterior arm. The long and lateral heads of the triceps muscle converged and fused to form this aponeurosis, which inserted into the posterior rough area of subcutaneous olecranon. The point of intersection (A) between the intramuscular septum with the TA was identified and labelled as 'point of confluence' (Fig. 1). Triceps splitting was done along the line of intramuscular septum to separate the long head from lateral head at the level two finger breadths proximal to the point of confluence. The point of intersection between RN within the spiral groove and mid axial humeral line was identified and marked as point ' $\mathrm{B}$. The distance between the point of confluence of TA (A) and the RN (point B) was measured and recorded as tricepso-radial distance (TRD) (Fig. 2). Furthermore,



Fig. 2. Showing the measurement of TRD distance between the point of confluence of TA (A) and the point of intersection of RN within spiral groove along the posterior aspect of the mid axial humerus (B). RN, radial nerve; TA, triceps aponeurosis; TRD, tricepso-radial distance. 
TA was also split to give better exposure for RN identification. $\mathrm{RN}$ was seen overlying the periosteum of the humerus within the spiral groove (Fig. 3). The distance of the RN from two bony landmarks, such as tip of acromion and tip of lateral epicondyle of humerus was also measured and recorded as acromio-radial distance (ARD) and condylo-radial distance (CRD) respectively. Measurements were taken with the help of surgical ruler. The distance from the tip of acromion to the tip of the lateral epicondyle of humerus was also measured and recorded as upper arm length (UAL). UAL represented the humeral length. Correlation analysis was done between UAL and all the measured distances for each cadaveric specimen.

Statistical analysis: Subsequent statistical analysis through paired, two-tailed Student $t$-test was performed (Microsoft Exel 2007; Microsoft, Redmond, WA, USA).

\section{Results}

In our study the mean value of UAL was $32.64 \pm 0.64 \mathrm{~cm}$.

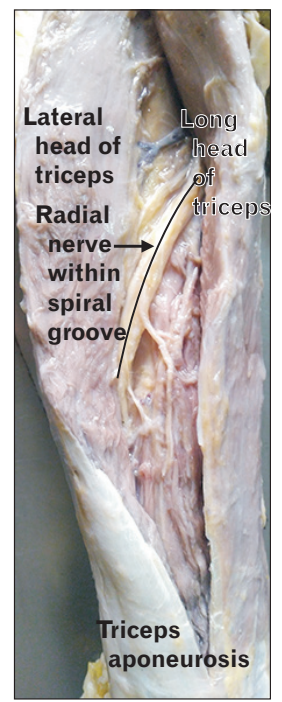

Fig. 3. Splitting of triceps aponeurosis for better exposure of radial nerve. The nerve can seen overlying the periosteum of the humerus and within the spiral groove.
The distance of the RN from the point of confluence of the TA (TRD), tip of the acromion (ARD) and tip of the lateral epicondyle of humerus (CRD) was $3.59 \pm 0.16 \mathrm{~cm}, 14.27 \pm 0.59$ $\mathrm{cm}$, and $17.14 \pm 1.29 \mathrm{~cm}$ respectively (Table 1 ). Statistically, TRD showed the least variability and CRD showed maximum variability (Fig. 4). The minimum TRD was found to be $3.00 \mathrm{~cm}$. So this should be considered as the maximum permissible length of the triceps split.

\section{Discussion}

Knowledge of the location of the RN and its relationship with a consistent and reliable anatomic landmark, are major concerns for treating surgeons.

Several studies have been done on various ethnic populations to determine the relationship between RN and bony landmarks, few of them have taken tip of lateral epicondyle of humerus as fixed bony point as in our case.

Guse et al. [15], Gerwin et al. [16], and Carlan et al. [11] have measured the distance between RN and lateral epicondyle of humerus in American population and found it to be $12.6 \pm 1.1 \mathrm{~cm}, 14.2 \pm 0.6 \mathrm{~cm}$, and $10.9 \pm 1.5 \mathrm{~cm}$ respectively.

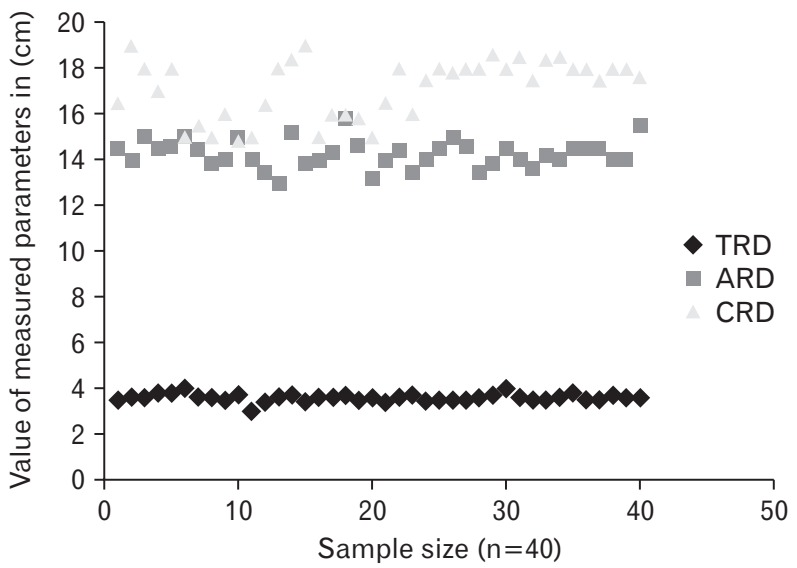

Fig. 4. Scatter plot showing distribution of measured parameters. ARD, acromion-radial distance; CRD, condylo-radial distance; TRD, tricepso-radial distance.

Table 1. Showing the values of the measured parameters

\begin{tabular}{ccccccc}
\hline Parameter & Mean $(\mathrm{cm})$ & SD & Variance & $95 \%$ CI & IQR & $P$-value \\
\hline UAL & 32.64 & 0.647413 & 0.41914414 & 0.200631 & 1 & 0.2 \\
TRD & 3.59 & 0.169161 & 0.02861538 & 0.052423 & 0.525 & \\
ARD & 14.27 & 0.599139 & 0.35896795 & 0.185672 & 2 & HS $(P<0.01)$ \\
CRD & 17.14 & 1.293762 & 1.67382051 & 0.400934 & 2 \\
\hline
\end{tabular}

SD, standard deviation; CI, confidence interval; IQR, interquartile range; UAL, upper arm length; TRD, tricepso-radial distance; ARD, acromion-radial distance; CRD, condylo-radial distance; HS, highly significant. 
In our study, on north Indian population, we found it to be $17.14 \pm 1.29 \mathrm{~cm}$.

Kamineni et al. [14] reported that the distance of the RN when it crosses the mid axial plane of humerus from the tip of lateral epicondyle of humerus was 1.4 to 2.0 times the trans epicondylar distance. On this basis, they proposed a 'safe zone' for external fixator pin entry into the lateral distal humerus.

Very few authors have located the $\mathrm{RN}$ in relation to the tip of acromion. Lusweti et al. [17] and Carlan et al. [11] have measured the distance between $\mathrm{RN}$ and tip of acromian (ARD) and found it to be $14.08 \pm 1.7 \mathrm{~cm}$ and $17.1 \pm 1.72$ $\mathrm{cm}$ respectively. In the present study, ARD was found to be $14.27 \pm 0.59 \mathrm{~cm}$.

All such studies observing relationships between $\mathrm{RN}$ and bony landmarks were conducted in cadaveric specimens having intact humerus; therefore, such anatomic relationships may not hold true in clinical situations. The relationships of the RN with various osseous landmarks did not have any correlative value, with wide inter-observer variability, and are difficult for surgeons to access intra operatively [8].

Based on cadaveric dissections, these studies show that the distance of the RN within the spiral groove from the tip of lateral epicondyle of humerus (CRD) ranges from $10 \mathrm{~cm}$ to $18 \mathrm{~cm}$. Whereas the distance of the RN from the tip of the acromion (ARD) ranges from $14 \mathrm{~cm}$ to $17 \mathrm{~cm}$. Considering the wide range of these proposed anatomic relationships, it may be difficult to localize the $\mathrm{RN}$ during the posterior approach to the humerus. Moreover, a surgeon is more interested in the relationship of the $\mathrm{RN}$ along the plane of surgical dissection (i.e., the intramuscular plane between the long and lateral head of triceps) rather than its relationship with the point where the $\mathrm{RN}$ enters or leaves the spiral groove. The exact localization of the RN using these bony points therefore seems to be questionable, and it may be imprudent for a surgeon to expect the existence of such relationships during operative fixation of fractures [18]. Therefore, in case of fracture and displacement of humerus, a non osseous superficial soft tissue landmark may guide the orthopaedician to identify the RN and prevent potential iatrogenic injury. There are few studies describing the relationship of the RN to soft tissue landmarks like the apex of the TA, triceps tendon and the point of confluence $[8,18-20]$. Among these, the point of confluence is an easily distinguishable and constantly present superficial landmark.

In the present study conducted on 40 upper extremities,
RN was consistently identified approximately two finger breadths proximal to the point of confluence with a mean of distance of $3.59 \pm 0.16 \mathrm{~cm}$.

Seigerman et al. [8] conducted a similar study on 30 adult human cadaveric upper extremities and reported the RN to lie consistently at distance of $39.0 \pm 2.1 \mathrm{~mm}$ proximal to the point of confluence.

Prasad et al. [20] studied 28 adult south Indian cadaveric arms and found the $\mathrm{RN}$ to lie $39.7 \pm 11.8 \mathrm{~mm}$ proximal to the point of confluence.

As evident from Table 1, the value of parameters of variability such as standard deviation, variance, $95 \%$ confidence interval of mean and Interquartile range were least for TRD. So, according to our findings, TRD can be considered the best measure to locate and protect the $\mathrm{RN}$ during surgery followed by ARD and CRD.

In conclusion, Soft tissue landmark (confluence of TA) is the most consistent and reliable to identify, locate and protect $\mathrm{RN}$ intraoperatively. The $\mathrm{RN}$ varies at different point within the spiral groove so it is always advised to take the shortest distance (TRD) as safe 'triceps split' by this way we can minimize the risk of nerve injury.

\section{ORCID}

Apurba Patra: https://orcid.org/0000-0002-1107-4481

Priti Chaudhary: https://orcid.org/0000-0002-5869-1743

Vishal Malhotra: https://orcid.org/0000-0002-9821-2284

Kamal Arora: https://orcid.org/0000-0002-6482-3685

\section{Author Contributions}

Conceptualization: AP, PC, KA. Data acquisition: AP, PC. Data analysis or interpretation: AP, VM. Drafting of the manuscript: AP, PC, VM. Critical revision of the manuscript: AP, PC, KA. Approval of the final version of the manuscript: all authors.

\section{Conflicts of Interest}

No potential conflict of interest relevant to this article was reported.

\section{References}

1. Fleming P, Lenehan B, Sankar R, Folan-Curran J, Curtin W. 
One-third, two-thirds: relationship of the radial nerve to the lateral intermuscular septum in the arm. Clin Anat 2004;17:269.

2. Standring S. Upper arm. In: Gray H, Standring S, Ellis H, Berkovitz BKB, editors. Gray's anatomy: the anatomical basis of clinical practice. 39th ed. Edinburgh: Elsevier Churchill Livingstone; 2005. p.851-8.

3. Mohler LR, Hanel DP. Closed fractures complicated by peripheral nerve injury. J Am Acad Orthop Surg 2006;14:32-7.

4. Shao YC, Harwood P, Grotz MR, Limb D, Giannoudis PV. Radial nerve palsy associated with fractures of the shaft of the humerus: a systematic review. J Bone Joint Surg Br 2005;87:164752.

5. Wang JP, Shen WJ, Chen WM, Huang CK, Shen YS, Chen TH. Iatrogenic radial nerve palsy after operative management of humeral shaft fractures. J Trauma 2009;66:800-3.

6. Hoppenfeld S, De Boer PG, Buckley R. Surgical exposures in orthopaedics: the anatomic approach. 4th ed. Philadelphia: Wolters Kluwer/Lippincott Williams \& Wilkins Health; 2009.

7. DeFranco MJ, Lawton JN. Radial nerve injuries associated with humeral fractures. J Hand Surg Am 2006;31:655-63.

8. Seigerman DA, Choung EW, Yoon RS, Lu M, Frank MA, Gaines LC, Liporace FA. Identification of the radial nerve during the posterior approach to the humerus: a cadaveric study. J Orthop Trauma 2012;26:226-8.

9. Artico M, Telera S, Tiengo C, Stecco C, Macchi V, Porzionato A, Vigato E, Parenti A, De Caro R. Surgical anatomy of the radial nerve at the elbow. Surg Radiol Anat 2009;31:101-6.

10. Bono CM, Grossman MG, Hochwald N, Tornetta P 3rd. Radial and axillary nerves. Anatomic considerations for humeral fixation. Clin Orthop Relat Res 2000;(373):259-64.
11. Carlan D, Pratt J, Patterson JM, Weiland AJ, Boyer MI, Gelberman RH. The radial nerve in the brachium: an anatomic study in human cadavers. J Hand Surg Am 2007;32:1177-82.

12. Chou PH, Shyu JF, Ma HL, Wang ST, Chen TH. Courses of the radial nerve differ between Chinese and Caucasians: clinical applications. Clin Orthop Relat Res 2008;466:135-8.

13. Cox CL, Riherd D, Tubbs RS, Bradley E, Lee DH. Predicting radial nerve location using palpable landmarks. Clin Anat 2010;23:420-6.

14. Kamineni S, Ankem H, Patten DK. Anatomic relationship of the radial nerve to the elbow joint: clinical implications of safe pin placement. Clin Anat 2009;22:684-8.

15. Guse TR, Ostrum RF. The surgical anatomy of the radial nerve around the humerus. Clin Orthop Relat Res 1995;(320):149-53.

16. Gerwin M, Hotchkiss RN, Weiland AJ. Alternative operative exposures of the posterior aspect of the humeral diaphysis with reference to the radial nerve. J Bone Joint Surg Am 1996;78:1690-5.

17. Lusweti V, Oluoch R, Ayumba BR, Njoroge A, Elbadawi MGY. The course of the radial nerve in relation to the humerus: a cadaveric study in a Kenyan adult population. East African Orthopaedic Journal 2019;13:20-5.

18. Arora S, Goel N, Cheema GS, Batra S, Maini L. A method to localize the radial nerve using the 'apex of triceps aponeurosis' as a landmark. Clin Orthop Relat Res 2011;469:2638-44.

19. Ashfaq Hasan S, Rauls RB, Cordell CL, Bailey MS, Nguyen T. "Zone of vulnerability" for radial nerve injury: anatomic study. J Surg Orthop Adv 2014;23:105-10.

20. Prasad M, Isaac M, Premkumar PS. Anatomic landmarks to identify the radial nerve during the posterior approach of the humerus: a cadaveric study. J Clin Diagn Res 2018;12:1-4. 